OPEN ACCESS

Edited by:

Oswin Grollmuss,

Université Paris-Sud, France

Reviewed by:

Ivan Zeljkovic,

Sisters of Charity Hospital, Croatia

Akbar Shafiee,

Tehran University of Medical

Sciences, Iran

*Correspondence:

Kaiyu Zhou

drkaiyuzhou@scu.edu.cn

Specialty section:

This article was submitted to

Pediatric Cardiology,

a section of the journal

Frontiers in Cardiovascular Medicine

Received: 08 June 2021

Accepted: 26 July 2021

Published: 23 August 2021

Citation:

Zhao L, Yan S, Wang T, Hua Y and Zhou K (2021) Case Report:

Wide-to-Narrow QRS Tachycardia in a

3-Month-Old Infant

Front. Cardiovasc. Med. 8:722376.

doi: 10.3389/fCvm.2021.722376

\section{Case Report: Wide-to-Narrow QRS Tachycardia in a 3-Month-Old Infant}

\author{
Liang Zhao ${ }^{1,2,3,4}$, Song Yan 1,2,3,4, Tao Wang ${ }^{1,2,3,4}$, Yimin Hua ${ }^{1,2,3,4}$ and Kaiyu Zhou ${ }^{1,2,3,4 *}$ \\ ${ }^{1}$ Department of Paediatric Cardiology, West China Second University Hospital, Sichuan University, Chengdu, China, ${ }^{2}$ The \\ Cardiac Development and Early Intervention Unit, West China Institute of Women and Children's Health, West China Second \\ University Hospital, Sichuan University, Chengdu, China, ${ }^{3}$ Key Laboratory of Birth Defects and Related Diseases of Women \\ and Children (Sichuan University), Ministry of Education, Chengdu, China, ${ }^{4}$ Key Laboratory of Development and Diseases of \\ Women and Children of Sichuan Province, West China Second University Hospital, Sichuan University, Chengdu, China
}

Introduction: It is rare to find that wide QRS tachycardia automatically changes to narrow QRS tachycardia, and it is more difficult to clarify the mechanism.

Case Report: A 3-month-old infant with recurrent paroxysmal supraventricular tachycardia underwent transesophageal cardiac electrophysiological examination. The wide QRS tachycardia was induced by atrial $\mathrm{RS}_{2}$ stimulation, and it soon changed to narrow QRS tachycardia automatically. By the accurate measurement of esophageal lead, it was found that the electrocardiogram changes completely conform to Coumel law. The mechanism of wide and narrow QRS tachycardia was orthodromic atrioventricular reentrant tachycardia with or without ipsilateral functional bundle branch block, and the accessory pathway was defined as the left free wall-concealed accessory pathway.

Conclusion: Transesophageal cardiac electrophysiological examination can reveal some special electrophysiological phenomena, and its non-invasive nature is especially suitable for infants.

Keywords: transesophageal, electrophysiological, tachycardia, infant, Coumel law

\section{INTRODUCTION}

Clinically, it is rare to find that wide QRS tachycardia automatically changes to narrow QRS tachycardia, and it is more difficult to clarify the mechanism. This article reports a case of a 3-month-old infant who was found to have the above-mentioned ECG changes during the transesophageal cardiac electrophysiological examination (TECEE). Through accurate measurement of the esophageal leads, we confirmed that the changes in the electrocardiogram conform to Coumel law, an important electrophysiological phenomenon, and clarified the mechanism of tachycardia.

\section{CASE PRESENTATION}

A 3-month-old infant, with a cough for 4 days and repeated paleness for 1 day, was admitted to the hospital. In the neonatal period, the infant was hospitalized in the neonatal department for sustained paroxysmal supraventricular tachycardia (SVT) and terminated with intravenous injection of adenosine triphosphate, and then followed by oral digoxin. After discharge, digoxin was discontinued because of the inadvisable opinion of the parent. The infant had a 6-year-old healthy brother and no history of tachycardia in his family. The mother of the infant was healthy 
during pregnancy, had no history of drug abuse, had no negative family background or events, and delivered at the age of 32 . Admission examination of the infant revealed general conditions of wellness, a heart rate of 128 beats/min (bpm) with normal heart sound, regular cardiac rhythm and no heart murmur, as well as warm extremities, no swelling of the liver, and no edema in the lower limbs, and no abnormal results in the following routine: electrocardiogram, echocardiography, myocardial injury and serum electrolyte. After the family members signed the informed consent form, the child underwent a transesophageal cardiac electrophysiological examination. Due to recurrent tachycardia, the child underwent TECEE after the informed consent was signed by the parents.

After intravenous injection of Midazolam $(0.2 \mathrm{mg} / \mathrm{kg})$, the patient was sedated. The $7 \mathrm{~F}$ esophageal electrode was inserted through the nose, and the DF-5A cardiac electrophysiological stimulation system was used for transesophageal atrial pacing.

The infant was stimulated by atrial $\mathrm{RS}_{2}$ program. When the program stimulus negative scan to the combined rhythm interval was $130 \mathrm{~ms}$, the $S_{2}-R$ interval was not significantly prolonged, and the wide QRS tachycardia of the left bundle branch block was induced at $259 \mathrm{bpm}$ (Figure 1). The QRS wave time limit was $115 \mathrm{~ms}$, the RP interval measured by the esophageal lead was fixed at $112 \mathrm{~ms}$, and the PR interval was $120 \mathrm{~ms}$. Sixteen seconds later, the tachycardia suddenly changed to a regular narrow QRS tachycardia of 306 bpm (Figure 2). The QRS wave time limit was $66 \mathrm{~ms}$, and the P wave was inverted in lead I. The esophageal lead measured the RP interval of $76 \mathrm{~ms}$ and the PR interval of $120 \mathrm{~ms}$. After capturing the atria with eight overspeed stimuli with a frequency of $350 \mathrm{bpm}$, the tachycardia suddenly stopped (Figure 3 ). There was no preexcitation pattern during sinus rhythm and atrial pacing.

During hospitalization, tachycardia did not occur again without special treatment. The infant was discharged after respiratory tract infection was cured. After discharge, the parents of the infant did not want him to take drugs for a long time to prevent tachycardia but required close follow-up. Once the tachycardia attack was found, it would be treated with drugs in time. After 3 months of follow-up, there was no recurrence of tachycardia.

\section{DISCUSSION}

Esophageal electrophysiological examination was adopted in this infant. Wide QRS tachycardia was unexpectedly induced during the programmed stimulation of atrial $\mathrm{RS}_{2}$, accompanied by restlessness in the infant. We needed to make a differential diagnosis immediately, especially to determine whether it was ventricular tachycardia or antidromic atrioventricular reentrant tachycardia, two wide QRS tachycardias that might quickly lead to hemodynamic instability. The ECG waveform of each lead was quickly observed. According to the four-step Brugada rule (1), the tachycardia did not have "absence of an RS complex in all precordial leads, no RS interval of the precordial lead more than $100 \mathrm{~min}$ " and there was no atrioventricular separation in the esophageal lead. According to the latest limb lead rule (LLA rule) (2), the tachycardia did not meet any of the following conditions: 1. monophasic $\mathrm{R}$ wave in lead aVR; 2. predominantly negative QRS in leads I, II, III; and 3. opposing QRS complex in the limb leads. In addition, the tachycardia was induced by atrial stimulation, and atrial stimulation rarely induced ventricular tachycardia. Therefore, the wide QRS complex tachycardia was insufficient evidence for ventricular tachycardia. Reviewing the induction process, there was no ECG waveform of ventricular preexcitation during the sinus rhythm and atrial $\mathrm{RS}_{2}$ program stimulation, so antidromic atrioventricular reentrant tachycardia could be basically ruled out.

Wide QRS complex tachycardia lasted $16 \mathrm{~s}$, it was too short to take therapy, and the wide QRS tachycardia suddenly turned into a regular, typical narrow QRS SVT. We were relieved and then SVT was stopped by the conventional method of $S_{1} S_{1}$ overspeed stimulation.

We probed into this process of wide and narrow QRS tachycardia carefully, and measured the esophageal lead accurately, and found that the tachycardia was completely accorded with the typical Coumel law, which was an important electrocardiographic phenomenon.

The ECG manifestations of Coumel law mainly include the following: 1. There are two types of QRS complex in patients with orthodromic atrioventricular reentrant tachycardia: narrow QRS complex (excitation normal descending ventricle) and wide QRS complex (excitation descending with the left or right bundle branch block). 2. It was suggested that the ipsilateral block bundle branch of the accessory pathway, the prolongation of the RR interval, was mainly the prolongation of the RP interval, but the $\mathrm{PR}$ interval remained the same when the RR interval with the bundle branch block was longer than that of narrow QRS wave $\mathrm{RR}$ interval $>35 \mathrm{~ms}$. It was suggested that the accessory pathway was located on the opposite side of the bundle branch block if the RR interval of the bundle branch block was equal to the RR interval of the narrow QRS complex.

After zooming in the figure and slowing down the paper speed to $50 \mathrm{~mm} / \mathrm{s}$, we accurately measured the ECG in the esophageal lead. It was found that the RP interval was $112 \mathrm{~ms}$ and the PR interval was $120 \mathrm{~ms}$ in the wide QRS tachycardia. In the narrow QRS tachycardia, the RP interval was $76 \mathrm{~ms}$ and the PR interval was $120 \mathrm{~ms}$. Both wide and narrow QRS tachycardia had the characteristics of RP interval less than the $\mathrm{PR}$ interval and RP interval was more than $70 \mathrm{~ms}$, which was consistent with atrioventricular reentrant tachycardia. Further analysis showed that from wide QRS tachycardia to narrow QRS tachycardia, RP interval was shortened by $36 \mathrm{~ms}$ (more than $35 \mathrm{~ms}$ ), while PR interval was completely unchanged. The change of QRS time limit was entirely due to the change of RP interval, which was in full compliance with Coumel law. Because Coumel law was only applicable to the bypass in the free wall of the left or right ventricle, and in the wide QRS tachycardia was a left bundle branch block pattern, the mechanism of wide and narrow QRS tachycardia was orthodromic atrioventricular reentrant tachycardia with or without the ipsilateral functional bundle branch block, and the accessory pathway was defined as the left free wall-concealed accessory pathway. 


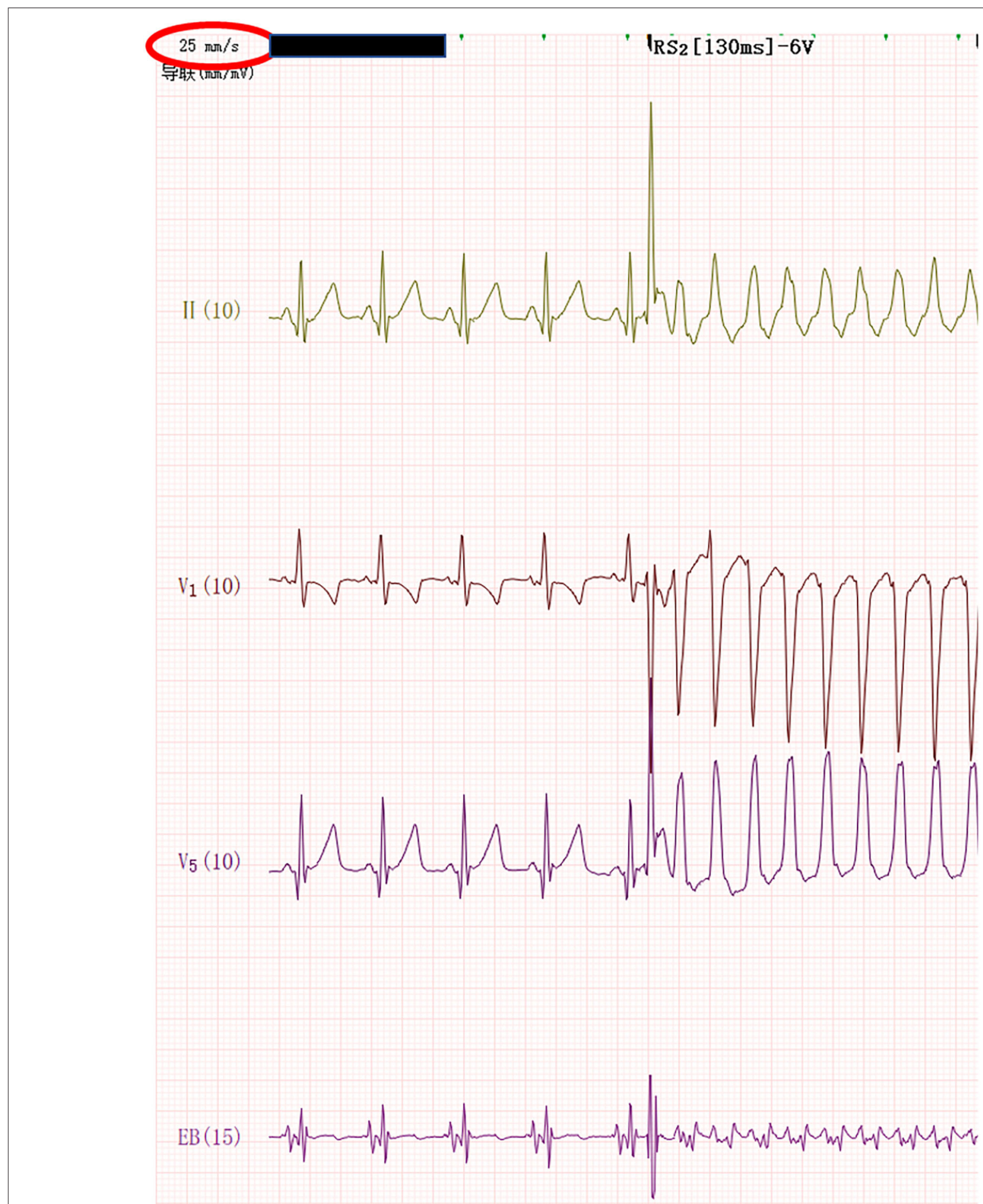

FIGURE 1 | When atrial $\mathrm{RS}_{2}$ program stimulation reached 130 min; the wide QRS tachycardia of the left bundle branch block was induced at 259 bpm. 


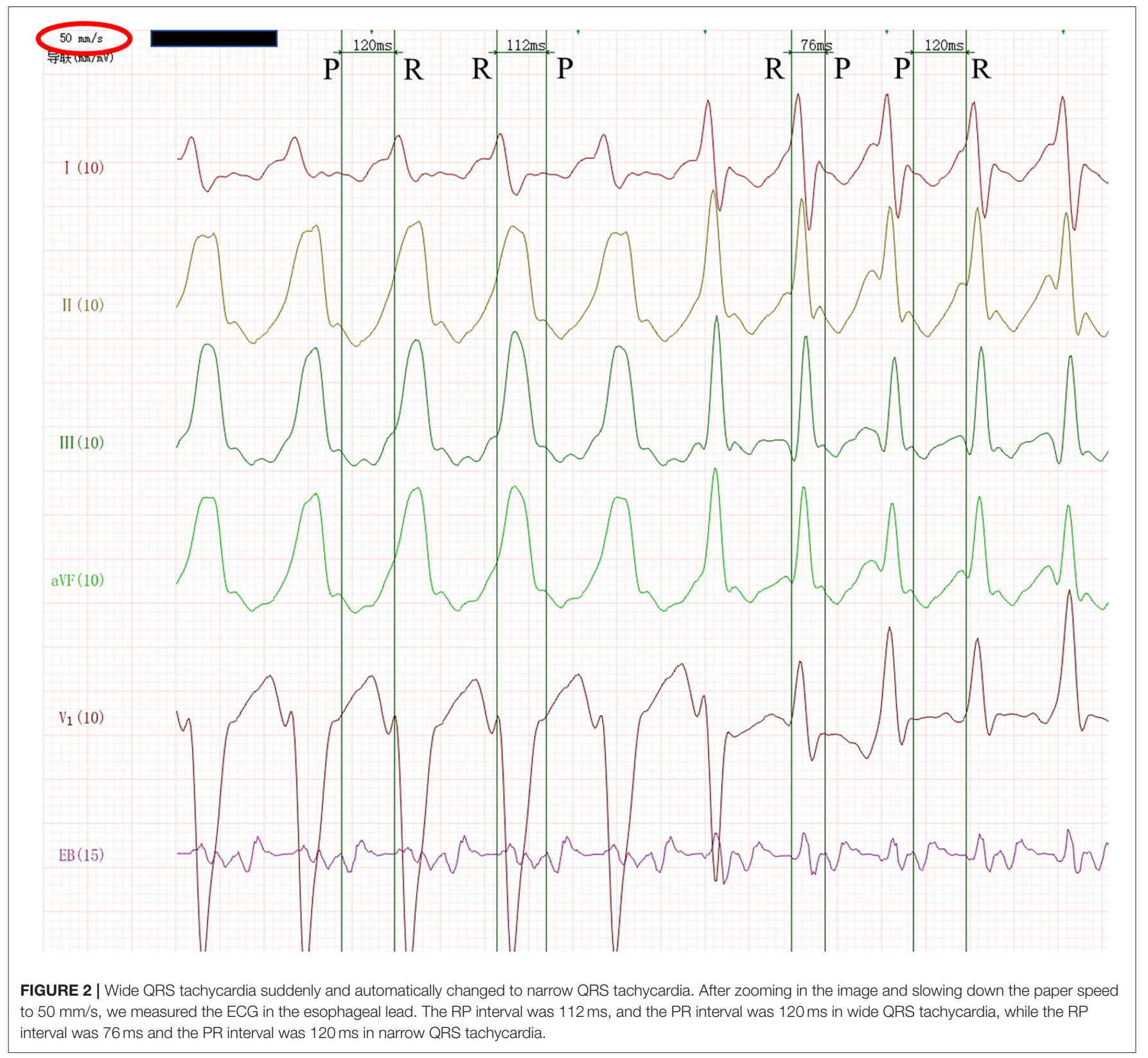

In this case, a non-invasive cardiac electrophysiological examination was performed for synchronous stimulation and recording. The process of inducing, changing, and terminating tachycardia and the characteristics of sudden abruptness were fully displayed, and typical figures conforming to Coumel law were collected. The RP and PR intervals of QRS complexes with different widths and narrowness were accurately measured so as to clarify the mechanism of tachycardia in the infant, locate the accessory pathway, and determine the direction for the future treatment of the infant.

Cardiac electrophysiological examination has important value for the determination of electrocardiographic phenomena $(3,4)$. Cardiac electrophysiological examination is divided into intracardiac electrophysiological examination and TECEE. The cases of wide QRS converted to narrow QRS tachycardia reported in the literature were mostly confirmed by intracardiac electrophysiological examination $(5,6)$. Compared with intracardiac electrophysiological examination, TECEE is simple, non-invasive, safe, inexpensive, and is especially suitable for infants who are temporarily unsuitable for radio frequency ablation.

TECEE can be carried out safely and effectively in infants and even newborns (7), which has important value in the diagnosis of supraventricular tachycardia and is worth promoting.

As far as we know, this is the first case report of automatic conversion of wide QRS to narrow QRS tachycardia in an infant, 


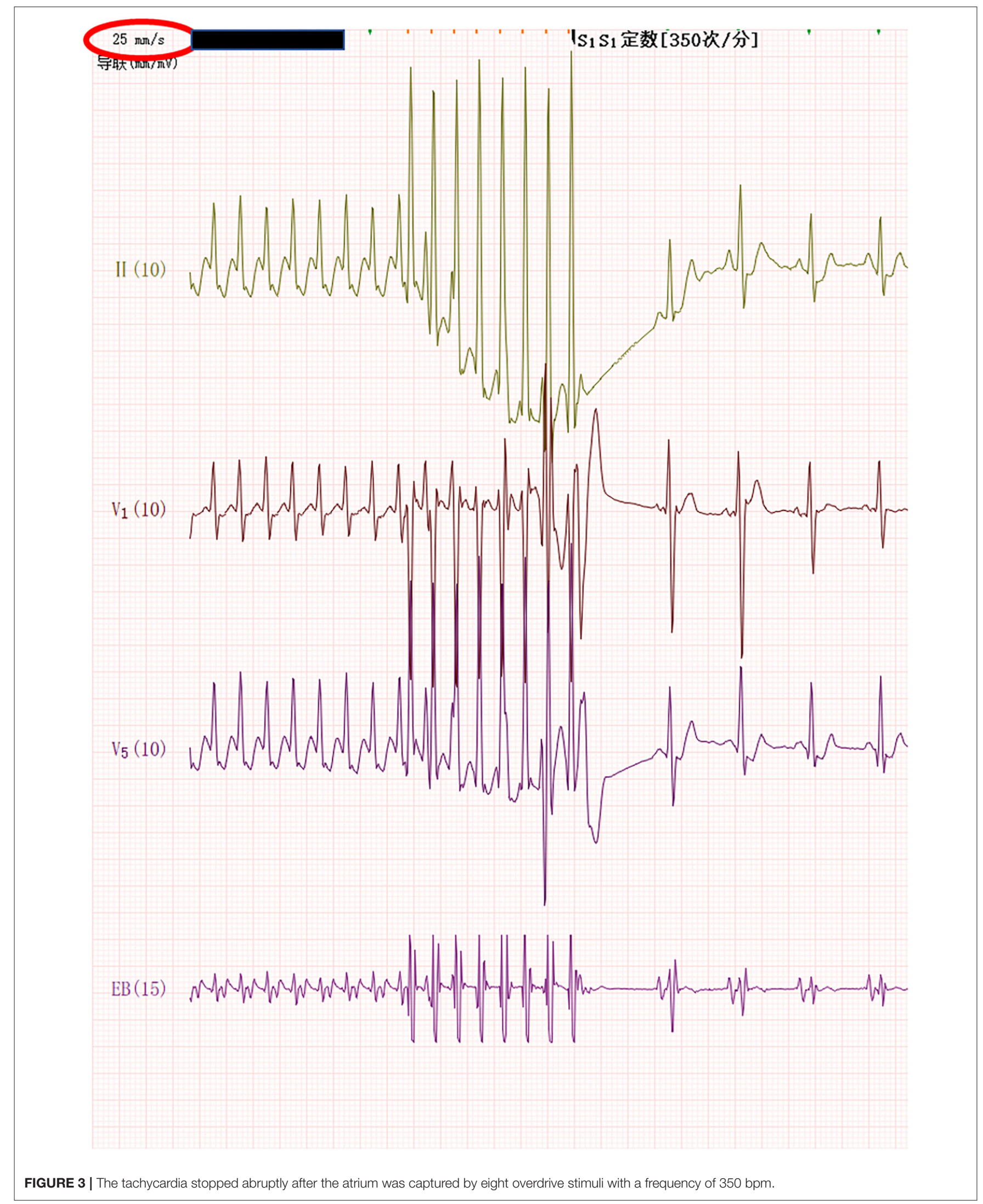


and the pathogenesis has been confirmed by the non-invasive examination method of TECEE.

\section{CONCLUSION}

Transesophageal cardiac electrophysiological examination can actively induce some special electrophysiological phenomena, which can be reasonably explained through the analysis of esophageal lead. Its non-invasive nature makes it particularly suitable for infants.

\section{DATA AVAILABILITY STATEMENT}

The original contributions presented in the study are included in the article/supplementary materials, further inquiries can be directed to the corresponding author/s.

\section{ETHICS STATEMENT}

The studies involving human participants were reviewed and approved by the Ethics Committee of West China Second University Hospital, Sichuan University. Written

\section{REFERENCES}

1. Brugada P, Brugada J, Mont L, Smeets J, Andries EW. A new approach to the differential diagnosis of a regular tachycardia with a wide QRS complex. Circulation. (1991) 83:1649-59. doi: 10.1161/01.CIR.83. 5.1649

2. Chen Q, Xu J, Gianni C, Trivedi C, Rocca DGD, Bassiouny M, et al. Simple electrocardiographic criteria for rapid identification of wide QRS complex tachycardia: the new limb lead algorithm. Heart Rhythm. (2020) 17:4318. doi: 10.1016/j.hrthm.2019.09.021

3. Otsuka N, Nagashima K, Wakamatsu Y, Okumura Y. Supraventricular tachycardia with QRS alternans: what is the mechanism? J Cardiovasc Electrophysiol. (2020) 31:1560-2. doi: 10.1111/jce.14508

4. Kara M, Korkmaz A, Karimli E, Rencuzogullari I, Ozeke O, Cay S. An unexpected ventriculoatrial block during the parahisian pacing maneuver and the retrograde gap phenomenon. J Cardiovasc Electrophysiol. (2020) 31:5501. doi: $10.1111 /$ jce.14334

5. Rohit M, Krishnappa D, Selvaraj RJ. Wide to narrow complex tachycardia: what is the diagnosis?J Cardiovasc Electrophysiol. (2018) 29:487-8. doi: 10.1111/jce.13417

6. Bohora S. Wide QRS tachycardia changing to a narrow QRS tachycardia. What is the mechanism? Heart Rhythm. (2015) 12:1698-700. doi: 10.1016/j.hrthm.2015.02.009 informed consent to participate in this study was provided by the participants' legal guardian/next of kin. Written informed consent was obtained from the minor(s)' legal guardian/next of kin for the publication of any potentially identifiable images or data included in this article.

\section{AUTHOR CONTRIBUTIONS}

$\mathrm{KZ}$ and $\mathrm{LZ}$ were responsible for the study design and manuscript preparation. SY, TW, and KZ were responsible for the clinical data. YH contributed to data acquisition. LZ wrote this manuscript. All authors contributed to the article and approved the submitted version.

\section{FUNDING}

This work was supported by key R \& D projects of Sichuan Science and Technology Department (Nos. 2019YFS0426, 2020YFS0101, and 2021YFS0094) and Scientific Research Project of Sichuan Health and Family Planning Commission (No. 17PJ260).

7. Chen M, Qin JH, Tan XL, Huang J, Ma LZ, Zhang XM, et al. Value of transesophageal atrial pacing in neonates with tachyarrhythmia. Zhongguo Dang Dai Er Ke Za Zhi. (2018) 20:734-6. doi: 10.7499/j.issn.1008-8830.2018.09.008

Conflict of Interest: The authors declare that the research was conducted in the absence of any commercial or financial relationships that could be construed as a potential conflict of interest.

Publisher's Note: All claims expressed in this article are solely those of the authors and do not necessarily represent those of their affiliated organizations, or those of the publisher, the editors and the reviewers. Any product that may be evaluated in this article, or claim that may be made by its manufacturer, is not guaranteed or endorsed by the publisher.

Copyright (c) 2021 Zhao, Yan, Wang, Hua and Zhou. This is an open-access article distributed under the terms of the Creative Commons Attribution License (CC BY). The use, distribution or reproduction in other forums is permitted, provided the original author(s) and the copyright owner(s) are credited and that the original publication in this journal is cited, in accordance with accepted academic practice. No use, distribution or reproduction is permitted which does not comply with these terms. 